INPLASY

PROTOCOL

To cite: Wang et al.

Association between ambient air pollution and in vitro fertilization outcomes: A system review and metaanalysis. Inplasy protocol 202140002. doi:

10.37766/inplasy2021.4.0002

Received: 31 March 2021

Published: 01 April 2021

Corresponding author:

Ruizhi Liu

liurz@jlu.edu.cn

Author Affiliation:

Center of Reproductive

Medicine and Center of

Prenatal Diagnosis, the First

Hospital, Jilin University

Support: None.

Review Stage at time of this submission: Preliminary

searches.

Conflicts of interest:

None declared.

\section{Association between ambient air pollution and in vitro fertilization outcomes: A system review and meta-analysis}

Wang, M¹; Yu, Y2; Wang, R3; Jiang, Y4; Zhang, H5; Liu, R6.

Review question / Objective: To explore the impact of exposure to ambient air pollution in different periods on the in vitro fertilization outcomes.

Condition being studied: In recent years, researches about the association between air pollutants and assisted reproduction outcomes has increased. The results of these studies have varied. And some scholars had conducted systematic reviews on the relationship between air pollution and infertility which also included the general population. The systematic review and meta-analysis that only focused on the association between air pollution and the outcomes of in vitro fertilization in infertility patients had not been studied.

Information sources: All studies were independently searched by two authors separately from the following databases: PubMed, EMBASE, Web of Science, and the Cochrane Library.

INPLASY registration number: This protocol was registered with the International Platform of Registered Systematic Review and Meta-Analysis Protocols (INPLASY) on 01 April 2021 and was last updated on 01 April 2021 (registration number INPLASY202140002).

\section{INTRODUCTION}

Review question / Objective: To explore the impact of exposure to ambient air pollution in different periods on the in vitro fertilization outcomes.
Condition being studied: In recent years, researches about the association between air pollutants and assisted reproduction outcomes has increased. The results of these studies have varied. And some scholars had conducted systematic reviews on the relationship between air 
pollution and infertility which also included the general population. The systematic review and meta-analysis that only focused on the association between air pollution and the outcomes of in vitro fertilization in infertility patients had not been studied.

\section{METHODS}

Participant or population: Infertile women who underwent fresh/frozen IVF cycles.

Intervention: IVF patients exposed to air pollutants. All pregnant women had a follow-up pregnancy outcome or a birth outcome follow-up.

Comparator: IVF patients exposed to air pollutants. All pregnant women did not have a follow-up pregnancy outcome or a birth outcome follow-up.

Study designs to be included: Retrospective (cohort) analyses.

Eligibility criteria: (1) Patients must have complete medical records and treatment records. (2) Studies explored the association between air pollutants (at least two or more) and IVF outcomes (at least one or more). (3) Air pollutants must be outdoor. (4) Studies on exposure to air pollution during the entire process of IVF treatment or at various periods of the treatment process. (5) Studies provided odds ratios (ORs) and their $95 \%$ confidence intervals (Cls) with per standard deviation (SD), interquartile range (IQR) or other specific unit change of the pollutants level.

Information sources: All studies were independently searched by two authors separately from the following databases: PubMed, EMBASE, Web of Science, and the Cochrane Library.

Main outcome(s): The association between ambient air pollution and in vitro fertilization outcomes.

Quality assessment / Risk of bias analysis: Two independent authors will carry out the quality assessment following the Newcastle-Ottawa Scale (NOS), and discordances will be discussed by a third reviewer and resolved by consensus.

Strategy of data synthesis: First, we will conduct a systematic review to achieve our study objective. Meta-analysis will be conducted if applicable (more than three studies). In the meta-analysis, the odd ratios (ORs) and $95 \%$ confidence intervals (CIs) were calculated using the inverse variance method to assess the ambient air pollutions and in vitro fertilization outcomes. The heterogeneity between studies was evaluated using $\mathbf{1 2}$ statistics. If


was moderate or low heterogeneity between studies and fixed effect model was adopted. Otherwise, a random effect model was applied ( $12 \geq 50 \%)$. Publication bias was evaluated by funnel plots and Egger tests, and p-values of Egger tests less than 0.05 showed there was publication bias. All the analyses were performed using STATA software (Version 13.0, Stata Corp, College Station, Texas, USA).

Subgroup analysis: If necessary, subgroup analyses of outcomes from different ages or different periods of IVF will be performed.

Sensitivity analysis: In order to get a more stable result, we may exclude studies with high risk of bias for sensitivity analysis.

Country(ies) involved: China, Korea, the United States, Brazil, France.

Keywords: ambient air pollution, PM2.5, PM10, NO2, in vitro fertilization, infertility.

Contributions of each author:

Author 1 - Mohan Wang.

Author 2 - Yang Yu.

Author 3 - Ruixue Wang.

Author 4 - Yuting Jiang.

Author 5 - Hongguo Zhang.

Author 6 - Ruizhi Liu. 